\title{
Фотолюминесценция с временны́м разрешением наноструктур InGaAs различной квантовой размерности
}

\author{
(C) А.М. Надточий ${ }^{1}$, С.А. Минтаиров ${ }^{2}$, Н.А. Калюжный ${ }^{2}$, М.В. Максимов ${ }^{1}$, \\ Д.А. Санников ${ }^{3,4}$, Т.Ф. Ягафаров ${ }^{3}$, А.Е. Жуков ${ }^{1}$ \\ ${ }^{1}$ Санкт-Петербургский национальный исследовательский Академический университет Российской академии наук, \\ 194021 Санкт-Петербург, Россия \\ ${ }^{2}$ Физико-технический институт им. А.Ф. Иоффре Российской академии наук, \\ 194021 Санкт-Петербург, Россия \\ ${ }^{3}$ Сколковский институт науки и технологий, \\ 121205 Москва, Россия \\ ${ }^{4}$ Физический институт им. П.Н. Лебедева Российской академии наук, \\ 119333 Москва, Россия \\ E-mail: al.nadtochy@mail.ioffe.ru
}

Поступила в Редакцию 22 мая 2019 г.

В окончательной редакции 27 мая 2019 г.

Принята к публикации 30 мая 2019 г.

\begin{abstract}
Методом время-коррелированного счета одиночных фотонов исследована фотолюминесценция с временны́ разрешением квантово-размерных гетероструктур InGaAs различной размерности, выращенных на подложках GaAs: квантовые точки, квантовые ямы и структуры переходной размерности — квантовые яма-точки. Обнаружено, что время спада фотолюминесценции образцов при комнатной температуре существенно зависит от их квантовой размерности: 6,7 и более 20 нс для квантовых точек, яма-точек и ямы соответственно. Мы полагаем, что наличие центров локализации носителей заряда может приводить к наблюдаемому сокращению времени фотолюминесценции в гетероструктурах.
\end{abstract}

Ключевые слова: фотолюминесценция, временно́е разрешение, квантово-размерные структуры.

DOI: $10.21883 /$ FTP.2019.11.48448.9167

\section{1. Введение}

Квантово-размерные структуры различной размерности, от квантовых ям (КЯ) - 2D до квантовых точек (KT) - 0D, находят успешное применение в различных оптоэлектронных приборах. Например, лазеры на КТ демонстрируют сверхнизкие пороговые токи [1], кроме того, излучатели на основе КТ низкой плотности нашли применение в качестве источников одиночных фотонов [2]; КЯ используются в качестве активной области мощных торцевые лазеров [3], поверхностноизлучающих лазеров на основе вертикального микрорезонатора. В последнее время проявляется значительный интерес к разработке квантово-размерных структур так называемой переходной размерности, например, проволок в яме (wires-on-well) [4], квантовых точек в нанопроволоках [5], яма-островковых структур (well-island composite structure) [6] или квантовых яма--точек (КЯТ, quantum well-dots) [7]. Последние из упомянутых структур могут быть представлены как сверхплотный массив КТ малого размера, или как КЯ с флуктуациями толщины и (или) состава. При этом такие структуры обладают рядом достоинств как КТ, так и КЯ и лишены некоторых их недостатков. Так, было показано, что максимальное поглощение/усиление на 1 слой КЯТ составляет более $50 \mathrm{~cm}^{-1}$ (для сравнения у слоя КТ около $\left.6-8 \mathrm{~cm}^{-1}[8]\right)$. Усиление в лазерах на КЯТ может быть дополнительно увеличено за счет синтеза большого количества слоев в активной области (более 15 слоев, см., например, [9]) даже без использования слоев компенсирующих упругие напряжения.

Благодаря таким уникальным свойства КЯТ удалось реализовать ряд приборов с рекордными характеристиками на их основе. В частности, КЯТ дают возможность существенно расширять диапазон спектральной чувствительности поглощения солнечных элементов на основе GaAs, что в перспективе позволит реализовать многопереходный фотопреобразователь со сбалансированными фототоками каскадов и, как следствие, повышенным кпд [9]. Использование структур с переходной размерностью в торцевых полупроводниковых лазерах позволяет получить как достаточно высокую выходную мощность излучения [10], так и широкий и однородный спектр усиления [6], что может быть востребовано в перестраиваемых лазерах. Кроме того, использование КЯТ перспективно в микролазерах, где необходим как высокий уровень усиления среды, так и подавленная латеральная подвижность носителей заряда. С использованием КЯТ были продемонстрированы наибольшая на сегодня температура лазерной генерации в микродисковых лазерах в непрерывном режиме $\left(110^{\circ} \mathrm{C}\right)$, а также рекордные значения оптической мощности и коэффициента полезного действия [11]. Была показана возможность прямой модуляции с частотой по крайней мере 6 ГГц, а минимальная энергия переключения составила 1.5 пДж/бит [12]. 
Интересным представляется вопрос влияния размерности активности среды на динамические свойства люминесценции. Так, например, в работе [2] авторы сравнивали массив КТ InAs/InGaAs/GaAs и КЯ InGAsN/GaAs диапазона 1.3 мкм и показали, что излучение КЯ спадает значительно быстрее $(\sim 0.15 \mathrm{Hc})$, чем у КТ $(\sim 1 \mathrm{Hc})$ в широком диапазоне температур.

В данной работе мы сравниваем особенности динамики фотолюминесценции (ФЛ) для наноструктур $\mathrm{In}(\mathrm{Ga}) \mathrm{As}$ различной квантовой размерности: ямы, точки и яма-точки.

\section{2. Эксперимент}

Исследования ФЛ в стационарном режиме были проведены с использованием лазера $\mathrm{YAG}: \mathrm{Nd}$ (532 нм) и охлаждаемого германиевого детектора в стандартной схеме синхронного детектирования. В качестве спектрального прибора использовался монохроматор МДР-23. Исследования время-разрешенной спектроскопии ФЛ были проведены с использованием метода время-коррелированного счета одиночных фотонов. Был использован конфокальный микроскоп, сопряженный с монохроматором (YobinYvon HR-320), лавинным фотодиодом (IDQ230), а также спектрометром Ocean optics QE-PRO. Для возбуждения использовался перестраиваемый лазер Ti : Sapphire (Coherent Chameleon Ultra II) с акустооптическим селектором импульсов. Площадь пятна возбуждения на образце на полувысоте составляла 230 мкм $^{2}$ и с сечением в форме распределения Гаусса. Возбуждающие импульсы с длиной волны в диапазоне 760-870 нм и длительностью 150 фс подавались с частотой 5-8 МГц, что позволяло исключить наложение последующих возбуждающих импульсов на фотолюминесценцию образцов. Мощность импульсов накачки варьировалась в диапазоне 10 мкВт-6 мВт (1 мВт с частотой 5 МГц и длиной волны 800 нм соответствует энергии

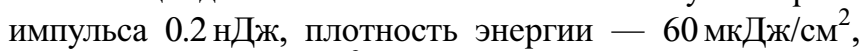
или $2.4 \cdot 10^{14}$ фотон $\left./ \mathrm{cm}^{2}\right)$. Для разделения возбуждающих импульсов и сигнала ФЛ использовались дихроичные зеркала (Thorlabs DMLP900 и Semrock TLP01-887). Синхронизация сигналов производилась с помощью модуля Becker\&Hick1 TCSPC160.

Для исследований были выбраны квантово-размерные структуры InGaAs различной размерности. Структуры, содержащие по одному слою КЯ (QW) и КЯТ (QWD), были выращены методом металлорганической газофазной эпитаксии на разориентированных на $6^{\circ}$ в направлении [111] подложках GaAs (100). Квантово-размерная активная область гетероструктур была окружена слоями $\mathrm{GaAs}$ толщиной 250-300 нм и барьерами $\mathrm{Al}_{0.3} \mathrm{Ga}_{0.7} \mathrm{As}$ толщиной 50 нм для предотвращения утечки носителей к поверхности и к подложке. КЯ представляла собой слой $\operatorname{In}_{0.2} \mathrm{Ga}_{0.8}$ As толщиной 8 нм. КЯТ были сформированы путем осаждения слоя $\mathrm{In}_{0.4} \mathrm{Ga}_{0.6} \mathrm{As}$ номинальной толщиной 8 монослоев (МС) [7].

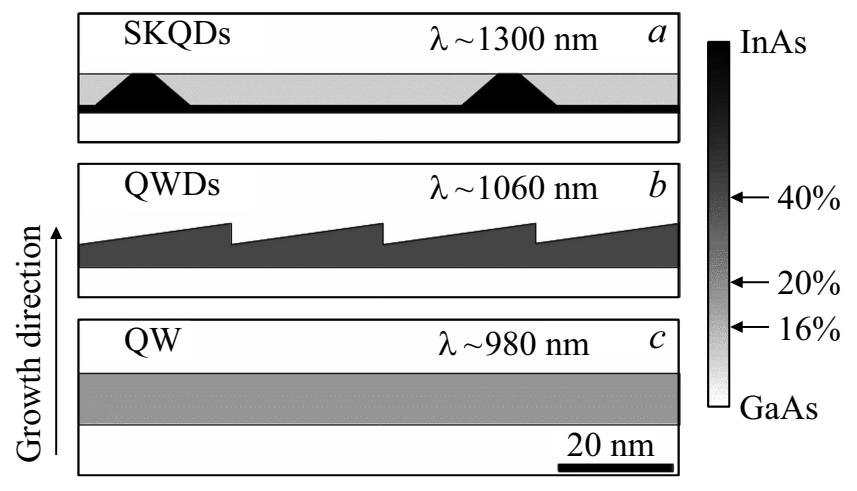

Рис. 1. Схематическое изображение сечения квантово-размерных областей исследуемых образцов: $a-$ КТ, $b-$ КЯТ, $c-\mathrm{KЯ)}$.

Структура с КТ, формируемыми по механизму Странского-Крастанова (SKQD), была выращена методом молекулярно-пучковой эпитаксии (МПЭ) на точно ориентированной подожке GaAs. Пять слоев КТ были равномерно распределены в слое GaAs толщиной 200 нм и окружены барьерами $\mathrm{Al}_{0.3} \mathrm{Ga}_{0.7} \mathrm{As}$ толщиной $20 \mathrm{HM}$. КТ были сформированы путем осаждения $2.6 \mathrm{MC}$ InAs с последующим заращиванием слоями $\operatorname{In}_{0.16} \mathrm{Ga}_{0.84} \mathrm{As}$ толщиной 5 нм.

Схематические изображения исследованных структур, подтвержденные полученными ранее данными просвечивающей электронной микроскопии, приведены на рис. 1. Массив КТ характеризуется плотностью $4-5 \cdot 10^{10} \mathrm{~cm}^{-2}$ на один слой островков InAs размером 15-18 нм и высотой 5 нм $[13,14]$ (рис. 1,a). Для слоев КЯТ характерны модуляции толщины слоя InGaAs на уровне 3-4 нм, имеющие поверхностную плотность $2-5 \cdot 10^{11} \mathrm{~cm}^{-2}[15,16]$ (рис. $\left.1, b\right)$. Структура с КЯ содержит однородный слой InGaAs толщиной 8 нм с гладкими гетероинтерфейсами (рис. 1,c).

\section{3. Результаты и обсуждение}

Спектры ФЛ исследованных структур, записанные в стационарном режиме при 78 и $300 \mathrm{~K}$ показаны на рис 2. Отметим, что спектры ФЛ при низких температурах, когда подавлен транспорт носителей заряда между локализующими центрами, отражают степень неоднородности структур, определяемой их квантовой размерностью. Спектр КТ при $78 \mathrm{~K}$ имеет ширину на полувысоте 40 мэВ, что обусловлено разбросом КТ по размерам. Ширина спектра ФЛ КЯТ и КЯ составляет 34 и 19 мэВ соответственно. Также отметим достаточно высокое оптическое совершенство всех образцов: соотношение интегральных интенсивностей при пониженной и комнатной температурах составляет 3.2, 1.6 и 1.3 для SKQD, QWD и QW соответственно. Таким образом, как по спектральной ширине линии, так и по стабильности 

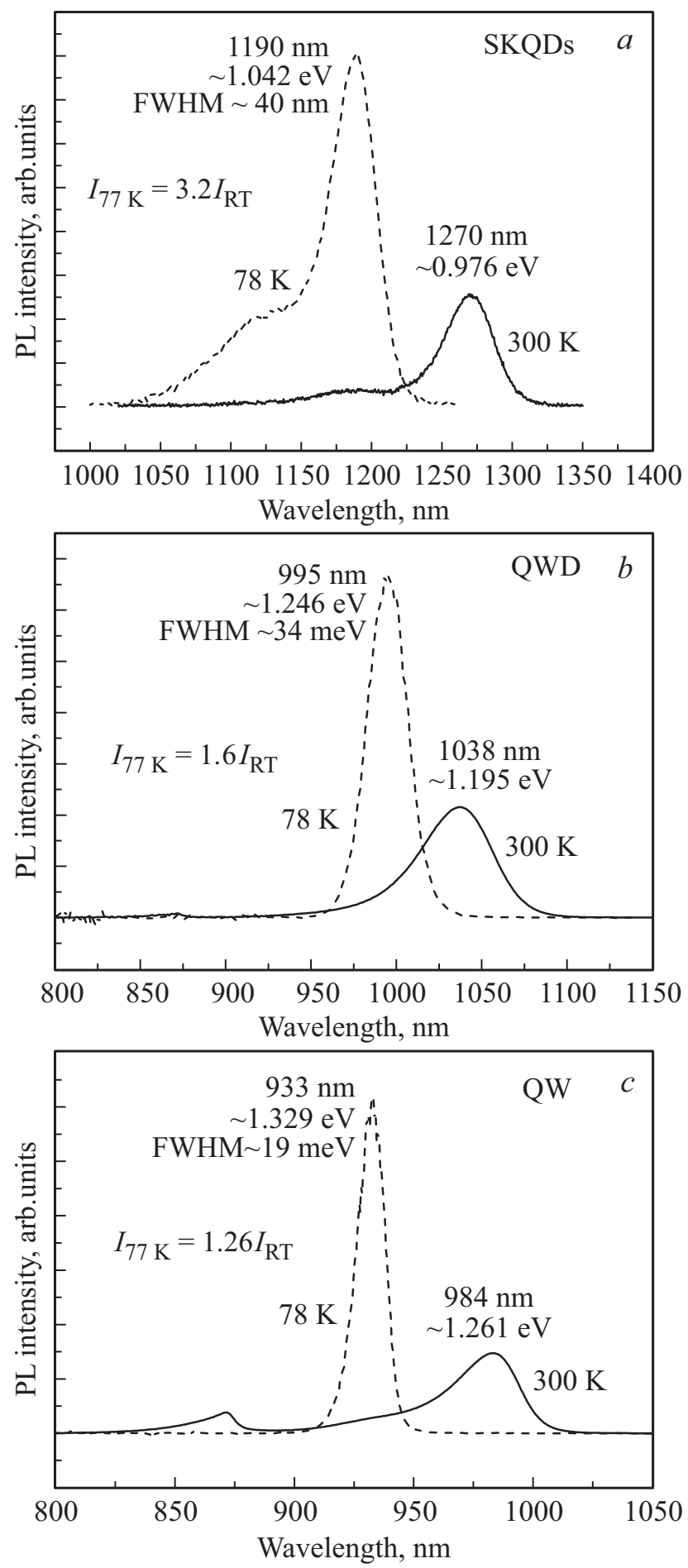

Рис. 2. Спектры ФЛ при непрерывном режиме возбуждения, записанные при температурах 78 и $300 \mathrm{~K}, a-\mathrm{KT}, b-$ КЯТ, $c$ - КЯ. Плотность мощности возбуждения $30 \mathrm{BT} / \mathrm{cm}^{2}$.

интенсивности, КЯТ занимают промежуточное положение между КТ и КЯ.

На рис. 3, а показана серия кривых, описывающих временну́ю эволюцию интенсивности сигнала ФЛ КТ на разных длинах волн. Видно, что с увеличением длины волны в пределах спектра излучения данного образца скорость спада ФЛ уменьшается, и в области пика основного состояния КТ можно наблюдать наиболее протяженный сигнал ФЛ. Можно выделить два характерных участка во временно́й кривой спада ФЛ, что особенно выражено в коротковолновой части спектра, и указывает на конкуренцию двух каналов рекомбинации. Полученные кривые спада ФЛ для всех длин волн хорошо аппроксимируются биэкспоненциальным выражением вида $\operatorname{PL}(t)=A_{1} \exp \left(\tau_{1} / t\right)+A_{2} \exp \left(\tau_{2} / t\right)$.

Таким образом, во временно́й зависимости ФЛ образца с КТ можно выделить быструю (время жизни 0.15-1.1 нс) и медленную компоненты (время жизни $2-7$ нс). Ранее многими группами $[17,18]$ для подобных КТ были получены значения времени жизни $\sim 1 \mathrm{Hc}$, причем, как правило, считается, что при комнатной
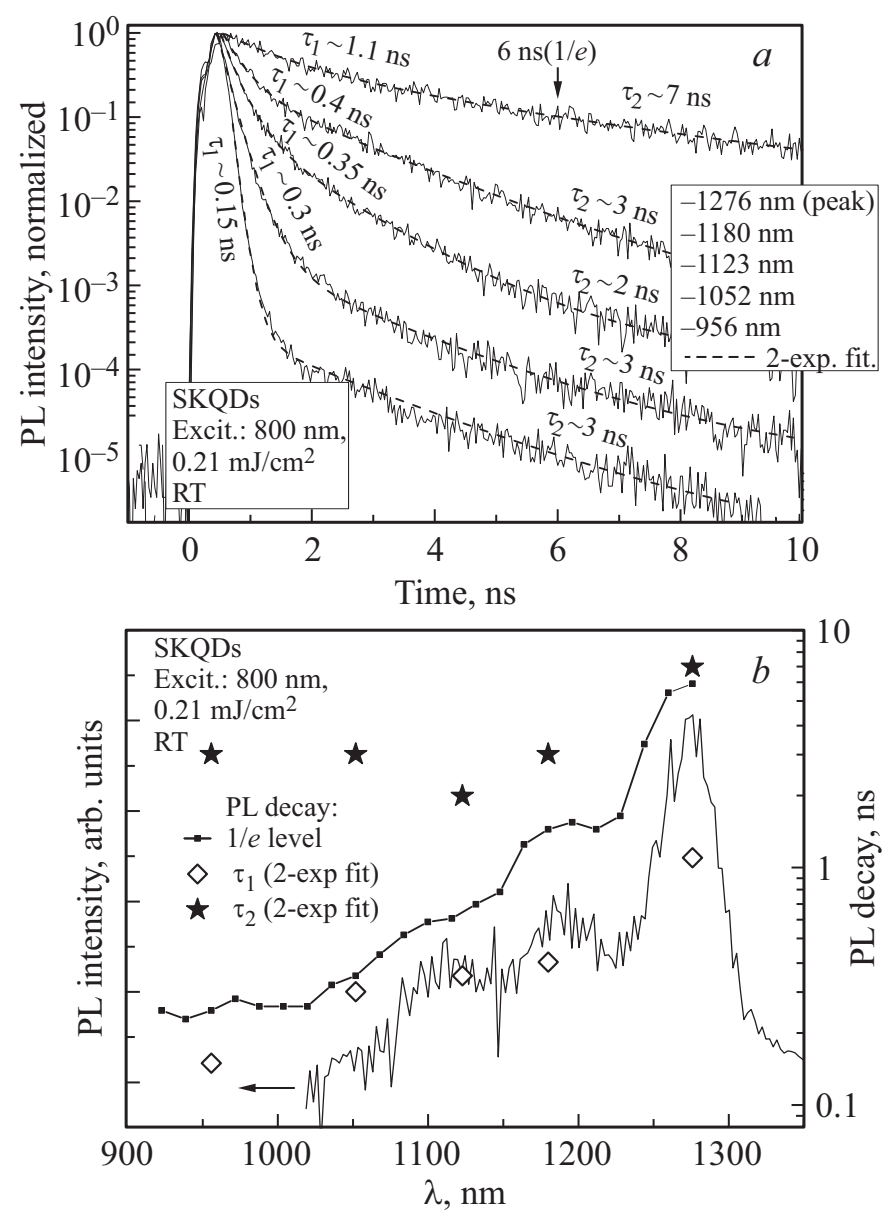

Рис. 3. $a-$ временна́я эволюция сигнала ФЛ на разных длинах волн и примеры аппроксимации полученных кривых выражением для двухканальной рекомбинации; $b-$ сопоставление спектра ФЛ и динамических характеристик ФЛ на разных длинах волн для структуры с КТ (кривая - время спада ФЛ по уровню $1 / e$; черные символы: ромб - быстрая компонента, звезда - медленная компонента, полученные при аппроксимации кривых спада ФЛ). Комнатная температура, длина волны возбуждения 800 нм и плотность энергии 0.21 мДж/см ${ }^{2}$ или $8 \cdot 10^{14}$ фотон $/ \mathrm{cm}^{2}$. 
температуре время жизни носителей заряда ограничено процессами безызлучательной рекомбинации [19].

Сопоставление спектра ФЛ структуры с КТ InAs/InGaAs/GaAs и спектра временны́х характеристик представлено на рис. $3, b$. Можно отметить, что спектр времени спада ФЛ приблизительно воспроизводит спектр ФЛ с наиболее длительной ФЛ в ее максимуме. При этом, если в области коротких длин волн спад ФЛ (по уровню $1 / e$ ) определяется быстрой компонентой и составляет $\sim 1 \mathrm{Hc}$, то в районе максимума ФЛ общий спад ФЛ определяется медленной компонентой и достигает 6 нс.

Спектральная зависимость времени жизни ФЛ определяется процессами релаксации носителей заряда. При возбуждении в матрицу GaAs носители заряда быстро релаксируют до уровней смачивающего слоя КТ ( 910-980нм), где на фоне более медленных процессов захвата на размерные уровни КТ образуется их значительная концентрация, сопровождающаяся интенсивной ФЛ с уровней смачивающего слоя. Далее происходит захват носителей заряда на уровни КТ, и интенсивность ФЛ смачивающего слоя спадает с характерным временем $\sim 1$ нс. Время спада ФЛ с уровней КТ при этом определяется медленной компонентой и составляет $\sim 7$ нс. Подобная интерпретация также рассматривалась в литературе (см., например, [17]), однако, как правило, полученные характерные времена были значительно короче (время релаксации/захвата носителей заряда $\sim 10-100$ пс, время жизни в КТ $\sim 1 \mathrm{Hc})$. Обнаруженное длинное время спада ФЛ может указывать на проявление таким образом эффекта перезахвата носителей заряда в массиве КТ, а также отсутствие в исследуемом образце каналов быстрой безызлучательной рекомбинации.

В работах [20,21], где также наблюдались быстрая и медленная компоненты в динамике ФЛ с основного состояния массива КТ, медленная компонента связывалась авторами с процессами транспорта и перезахвата носителей между КТ и имела характерное время 50-100 нс. Быстрая компонента ФЛ, измеренная при температуре $77 \mathrm{~K}$ и характеризующая излучательное время жизни носителей заряда в КТ, в зависимости от условий роста гетероструктуры и возбуждения ФЛ варьировалась в диапазоне $0.8-5.3$ нс. Это было объяснено особенностями заполнения массива КТ: при ма-

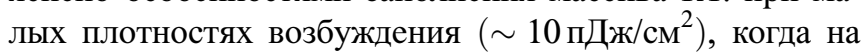
одну КТ приходится менее одной электронно-дырочной пары, возрастает вероятность заполнения КТ носителями только одного знака, что приводит к увеличению быстрой компоненты излучательного время жизни (свыше 5 нс). С увеличением плотности мощности возбужде-

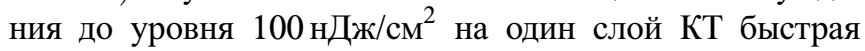
компонента излучательного времени жизни уменьшалась до 2 нс.

Спектр ФЛ и спектральное распределение времени спада ФЛ структур с КЯТ по уровню $1 /$ е при трех мощностях возбуждения (длина волны 870 нм) представлены
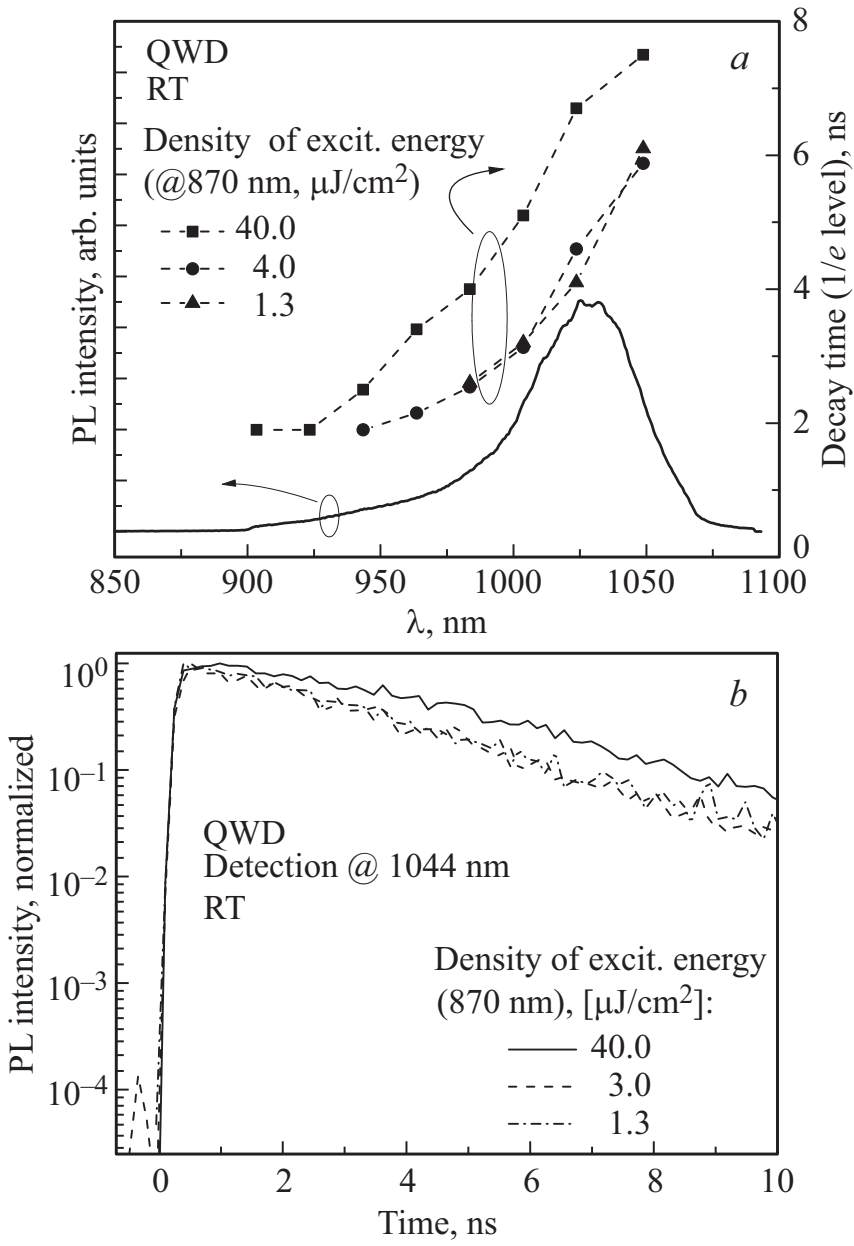

Рис. 4. $a$ - спектральное распределение ФЛ и времени спада ФЛ по уровню „е “ при некоторых мощностях возбуждения для структуры с КЯТ при комнатной температуре; $b$ - временны́е зависимости ФЛ после импульсного возбуждения с различной мощностью возбуждения при детектировании на длине волны 1044 нм.

на рис. 4 , а. Примеры временно́го распределения сигнала ФЛ на длине волны 1044 нм (соответствует наиболее длительной ФЛ) приведены на рис. $4, b$. С увеличением длины волны в пределах спектра излучения данного образца скорость спада ФЛ уменьшается, при этом максимальное время спада ФЛ незначительно растет с ростом уровня накачки и составляет 6-8 нс. Также можно отметить, что временны́е зависимости ФЛ достаточно хорошо аппроксимируются моноэкспонентной функцией, однако с ростом накачки наблюдается слабое проявление ,Плато“ в сигнале ФЛ (в диапазоне шкалы времени 4-6 нс). Феномен такого плато во временно́й зависимости ФЛ при возбуждении выше уровня матрицы GaAs наблюдался, например, в работе [22], где был интерпретирован авторами как проявление медленных механизмов релаксации носителей заряда на размерные уровни КТ (при резонансном возбуждении плато отсутствует). Мы полагаем, что отличие в динамике ФЛ 

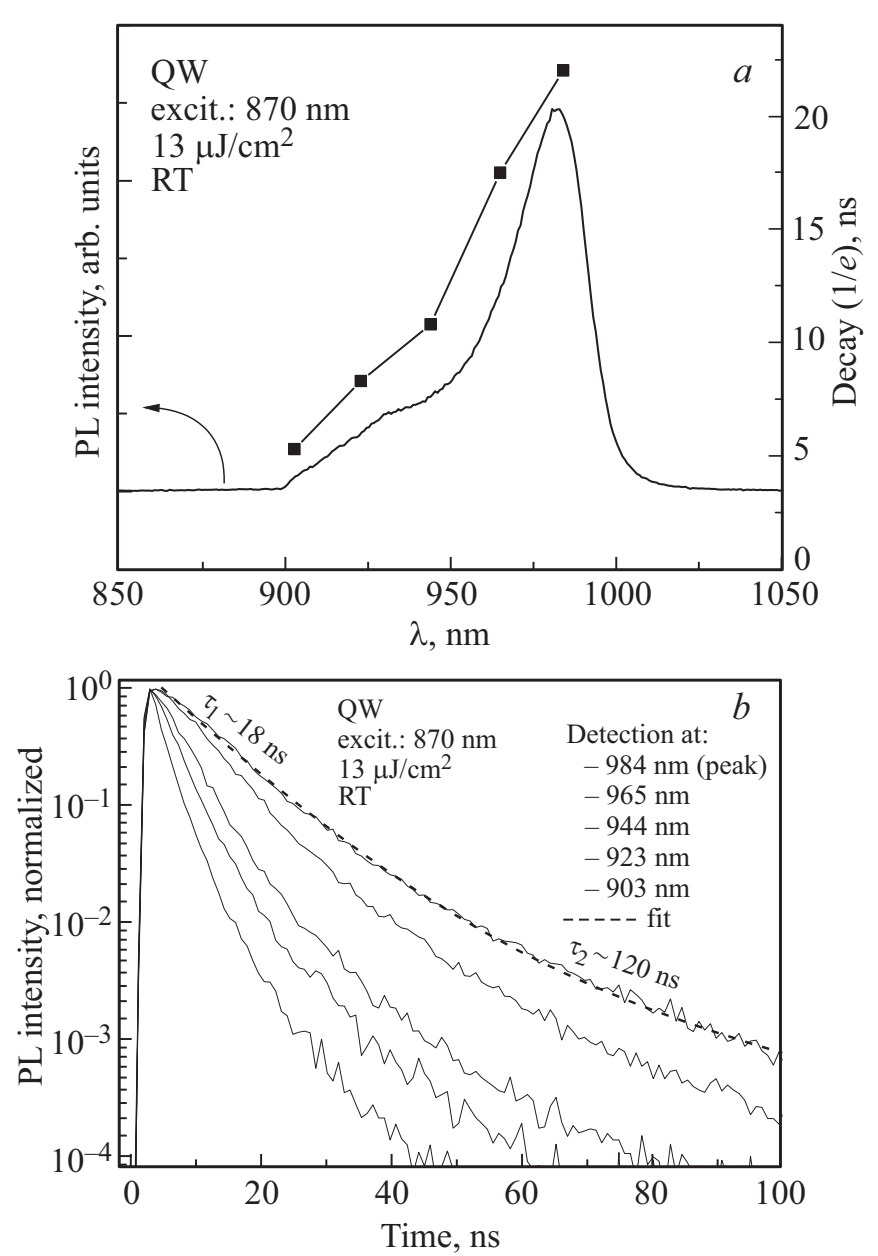

Рис. 5. $a-$ спектральное распределение ФЛ и времени спада ФЛ по уровню $1 / e$ для структуры с КЯ при комнатной температуре и мощности возбуждения 13 мкДж/см²; $b-$ временны́е зависимости ФЛ после импульсного возбуждения на различных длинах волн.

структур КЯТ и КТ может быть связано с отсутствием у первых промежуточных возбужденных состояний, участвующих в захвате носителей на более низкие энергетические уровни.

На рис. 5 представлены временны́е зависимости ФЛ

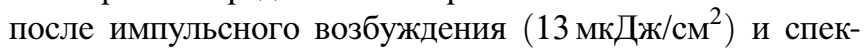
тральные зависимости ФЛ и спада ФЛ по уровню $1 / e$ для структуры с КЯ, которая демонстрирует относительно медленное затухание ФЛ: в максимуме ФЛ характерное время спада составляет $\sim 20$ нс. Так же как и в образцах с КТ и КЯТ, с ростом длины волны скорость спада ФЛ уменьшается. Вблизи основного состояния КЯ в ФЛ проявляется медленная компонента с характерным временем 120 нс. По аналогии с [20,21], где большие времена спада ФЛ объяснены перезахватом носителей между КТ в массиве, можно предположить наличие в структуре неких ловушек - неизлучающих центров локализации носителей заряда, медленный захват и выброс из которых может проявляться подобным образом.
На рис. 6, а представлено сопоставление спектров ФЛ и времени спада ФЛ по уровню $1 / e$ для двух условий возбуждения. Спектр ФЛ при повышении накачки существенно изменяется - происходит насыщение основного уровня КЯ и увеличение сигнала с возбужденных уровней (резкое падение сигнала на длинах волн короче 900 нм обусловлено дихроичнным фильтром DMLP900). Время спада ФЛ с увеличением накачки незначительно увеличивается, сохраняя свое спектральное распределение. На зависимостях ФЛ от времени (рис. 6, $b$ ) можно отметить несколько ключевых особенностей. Во-первых, не наблюдается сверхдлинной временно́й составляющей ФЛ (как при меньшей плотности возбуждения на рис. $5, b)$ - ФЛ в максимуме спадает моноэкспоненциально с характерным временем $\sim 20$ нс. Во-вторых, можно отметить длинное плато и значительную длительность $(\sim 3$ нс) времени возрастания сигнала на временно́й зависимости ФЛ в пике сигнала. В то же время на более коротковолновых кривых спада ФЛ можно отметить быстрое (характерное время $\sim 1.5 \mathrm{Hc}$
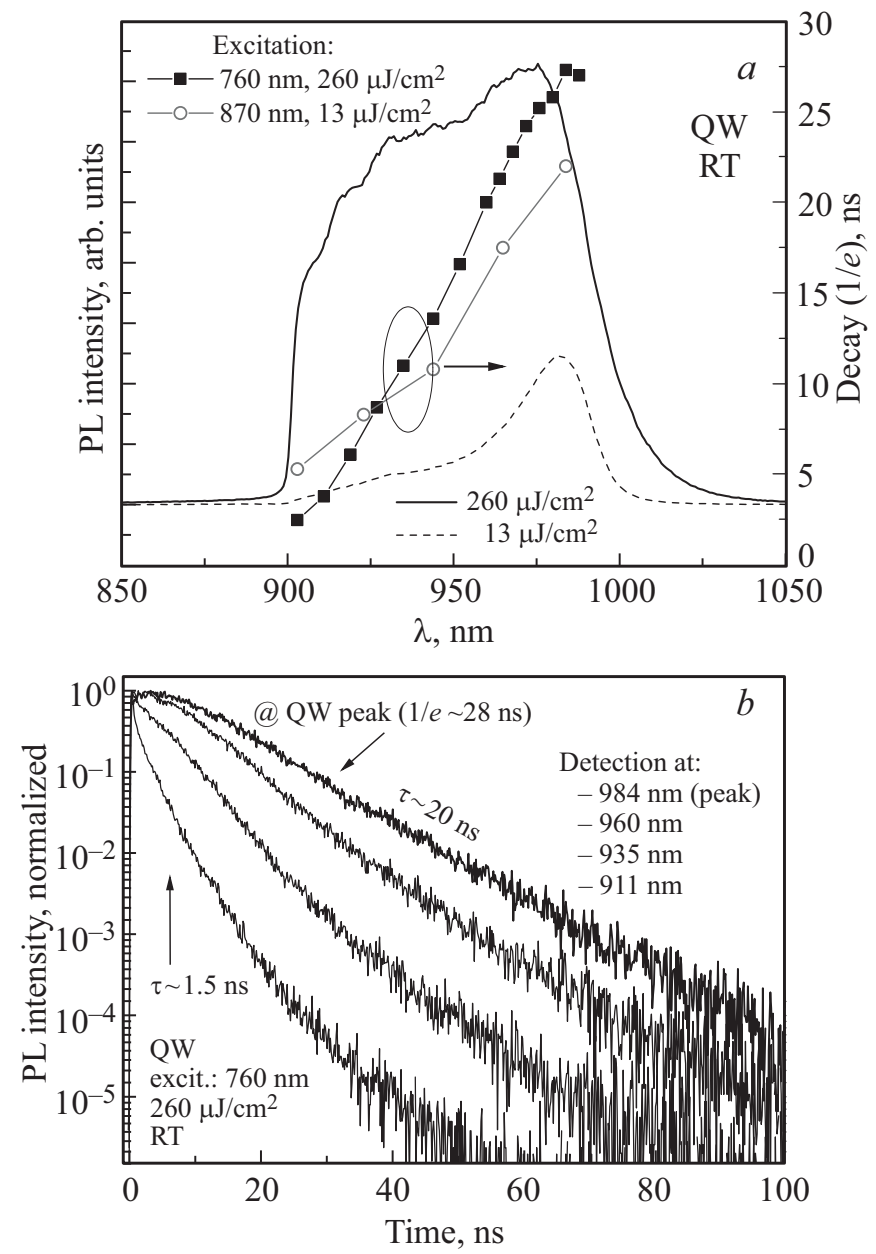

Рис. 6. $a-$ спектральное распределение ФЛ и времени спада ФЛ по уровню $1 / e$ при различных мощностях возбуждения для структуры с КЯ при комнатной температуре, мощности возбуждения 260 мкДж/см ${ }^{2} ; b-$ временнб́е зависимости ФЛ после импульсного возбуждения на различных длинах волн. 
на длине волны 911 нм) падение сигнала ФЛ. Такое поведение может иллюстрировать процессы релаксации носителей заряда: в начальный период $(0-3$ нс) высокая концентрация, сопровождающаяся интенсивной ФЛ, образуется на возбужденных состояниях КЯ, а на основном состоянии концентрация носителей заряда и, соответственно, сигнал ФЛ ниже. Далее происходит релаксация носителей с возбужденных уровней КЯ на основное состояние с характерным временем $1.5 \mathrm{Hc}$, и сигнал ФЛ на коротких длинах волн значительно снижается, а на основном состоянии растет с аналогичным характерным временем. Далее происходит постепенная рекомбинация носителей в КЯ на основном состоянии. Необычным, однако, являются большие времена наблюдаемых процессов: как правило, релаксация и рекомбинация носителей заряда в структурах с КЯ происходят значительно быстрее - релаксация 10-100пс, рекомбинация 0.1-1 нс (см., например, [18]).

Таким образом, полученные нами результаты показывают, что время спада ФЛ при комнатной температуре последовательно увеличивается с изменением квантовой размерности структур: $1-7$ нс для КТ, 6-8 нс для КЯТ, 20 нс для КЯ. При этом в случае массива КТ полученные результаты находятся в хорошем согласии с ранее публиковавшимися значениями для аналогичных структур. Однако полученное время спада для КЯ выглядит весьма необычно для комнатной температуры.

Время спада ФЛ, как правило, с температурой ведет себя немонотонно вследствие конкуренции механизмов излучательной и безызлучательной рекомбинации. При этом максимальные значения длительности спада ФЛ для КЯ, сопоставимые с полученными нами, лежат в диапазоне температур 150-250 К и ограничены временем излучательной рекомбинации [23]. При повышении температуры фотолюминесцентное время жизни, ограниченное процессами безызлучательной рекомбинации, быстро спадает. Учитывая превосходное оптическое качество образцов, особенно в случае КЯ, где интегральная интенсивность ФЛ при комнатной температуре сопоставима со значениями при $78 \mathrm{~K}$ (для сравнения, в [23] падение интенсивности ФЛ от 10 до $250 \mathrm{~K}$ составило 2 порядка!), можно предположить, что темп процессов безызлучательной рекомбинации несуществен даже при комнатной температуре, и время жизни носителей заряда ограничено временем излучательной рекомбинации. Если предположить, что это верно для всех исследованных образцов (так как все три образца показали превосходную температурную стабильность), то значения времени спада ФЛ соответствуют излучательному времени жизни носителей заряда.

Таким образом, представленные данные показывают сокращение времени излучательной рекомбинации носителей заряда при уменьшении квантовой размерности $\left(\tau_{S K Q D}<\tau_{Q W D}<\tau_{Q W}\right)$. Это может быть следствием влияния центров локализации носителей заряда на динамику излучательной рекомбинации: глубокая локализация носителя заряда какого-либо знака приведет к появлению нескомпенсированного заряда в КТ и может увеличить вероятность притяжения носителя другого знака.

Для надежного подтверждения высказанных предположений мы планируем провести детальные исследования ФЛ с временны́м разрешением в широком диапазоне температур, что позволит разделить излучательную и безызлучательную составляющие времени жизни носителей заряда.

\section{4. Заключение}

Методом ФЛ с временны́м разрешением были исследованы гетероструктуры различной квантовой размерности: квантовые точки, квантовые ямы и гибридные структуры переходной размерности - квантовые яма-точки. Обнаруженная динамика ФЛ для этих структур показала, что время спада фотолюминесценции в структурах с квантовыми точками, т.е. ярко выраженными центрами локализации носителей заряда, значительно короче, чем в структурах с переходной размерностью (квантовых яма-точках), в которых энергия локализации носителей значительно меньше и тем более, чем в квантовых ямах, где латеральная локализация носителей отсутствует.

Мы ожидаем, что исследования ФЛ с временны́м разрешением при пониженных температурах, а также с резонансным возбуждением позволят глубже понять физические процессы в исследованных структурах.

\section{Финансирование работы}

Работа выполнена при поддержке гранта РНФ № 16-12-10269.

\section{Конфликт интересов}

Авторы статьи заявляют, что у них нет конфликта интересов.

\section{Список литературы}

[1] S. Freisem, G. Ozgur, K. Shavritranuruk, H. Chen, D.G. Deppe. Electron. Lett., 44, 679 (2008).

[2] C. Carmesin, F. Olbrich, T. Mehrtens, M. Florian, S. Michael, S. Schreier, C. Nawrath, M. Paul, J. Höschele, B. Gerken, J. Kettler, S.L. Portalupi, M. Jetter, P. Michler, A. Rosenauer, F. Jahnke. Phys. Rev. B, 98, 125407 (2018).

[3] P. Crump, G. Blume, K. Paschke, R. Staske, A. Pietrzak, U. Zeimer, S. Einfeldt, A. Ginolas, F. Bugge, K. Häusler, P. Ressel, H. Wenzel, G. Erbert. Proc. SPIE, 7198, 719814 (2009).

[4] M. Sugiyama, H. Sugiyama, T. Katoh, K. Toprasertpong, H. Sodabanlu, K. Watanabe, D. Alonso-Alvarez, N.J. EkinsDaukes, Y. Nakano. Prog. Photovolt.: Res. Appl., 24, 1606 (2016). 
[5] G.E. Cirlin, R.R. Reznik, I.V. Shtrom, A.I. Khrebtov, Yu.B. Samsonenko, S.A. Kukushkin, T. Kasama, N. Akopian, L. Leonardo. Semiconductors, 52 (4), 462 (2018).

[6] Qingnan Yu, Xue Li, Yan Jia, Wei Lu, Ming Zheng, Xing Zhang, Yongqiang Ning, Jian Wu. ACS Photonics, 5 (12), 4896 (2018).

[7] S.A. Mintairov, N.A. Kalyuzhnyy, V.M. Lantratov, M.V. Maximov, A.M. Nadtochiy, S. Rouvimov, A.E Zhukov. Nanotechnology, 26, 385202 (2015).

[8] А.Е. Жуков, М.В. Максимов, А.Р. Ковш. ФТП, 46 (10), 1249 (2012).

[9] S.A. Mintairov, N.A. Kalyuzhnyy, M.V. Maximov, A.M. Nadtochiy, A.E. Zhukov. Semicond. Sci. Technol., 32, 015006 (2017).

[10] A.S. Payusov, Yu.M. Shernyakov, A.A. Serin, A.M. Nadtochiy, S.A. Mintairov, N.A. Kalyuzhnyy, M.M. Kulagina, A.E. Zhukov, N.Yu. Gordeev, M.V. Maximov. IOP Conf. Ser.: J. Physics: Conf. Ser., 1135, 012071 (2018).

[11] E. Moiseev, N. Kryzhanovskaya, M. Maximov, F. Zubov, A. Nadtochiy, M. Kulagina, Yu. Zadiranov, N. Kalyuzhnyy, S. Mintairov, A. Zhukov. Optics Lett., 43, 4554 (2018).

[12] A.E. Zhukov, N.V. Kryzhanovskaya, M.V. Maximov. Int. Conf. „Frontiers of 21st Century Physics and Ioffe Institute“ (St. Petersburg, Russia, 2018) p. 33.

[13] Б.В. Воловик, А.Ф. Цацульников, Д.А. Бедарев, А.Ю. Егоров, А.Е. Жуков, А.Р. Ковш, Н.Н. Леденцов, М.В. Максимов, Н.А. Малеев, Ю.Г. Мусихин, А.А. Суворова, В.М. Устинов, П.С. Копьев, Ж.И. Алфёров, Д. Бимберг, П. Веренер. ФТП, 33 (8), 990 (1999).

[14] M. Gutiérrez. Proc. SPIE, 5840, 486 (2005).

[15] A.M. Nadtochiy, M.V. Maximov, S.A. Mintairov, N.A. Kalyuzhnyy, Y.M. Shernyakov, A.S. Payusov, A.E. Zhukov, S. Rouvimov, A.V. Savelyev. Proc. SPIE, 10114, UNSP 101140Y (2017).

[16] А.М. Надточий, С.А. Минтаиров, Н.А. Калюжный, С.С. Рувимов, Ю.М. Шерняков, А.С. Паюсов, М.В. Максимов, А.Е. Жуков. ФТП, 49 (8), 1115 (2015).

[17] R. Heitz, H. Born, T. Luttgert, A. Hoffmann, D. Bimberg. Phys. Status Solidi B, 221, 65 (2000).

[18] A. Markus, A. Fiore, J.D. Ganiere, U. Oesterle, J.X. Chen, B. Deveaud, M. Ilegems, H. Reichert. Appl. Phys. Lett., 80 (6), 911 (2002).

[19] O. Nasr, N. Chauvin, M.H. Hadj Alouane, H. Maaref, C. Bru-Chevallier, L. Sfaxi, B. Ilahi. J. Opt., 19, 025401 (2017).

[20] L.Ya. Karachinsky, S. Pellegrini, G.S. Buller, A.S. Shkolnik, N.Yu. Gordeev, V.P. Evtikhiev, V.B. Novikov. Appl. Phys. Lett., 84 (1), 7 (2004).

[21] S. Pellegrini, G.S. Buller, L.Ya. Karachinsky, A.S. Shkolnik, N.Yu. Gordeev, G.G. Zegrya, V.P. Evtikhiev, I.R. Sellers, M.S. Skolnick, H.Y. Liu, M. Hopkinson. Proc. SPIE, 5725, 309 (2005).

[22] I. Gontijo, G.S. Buller, J.S. Massa, A.C. Walker, S.V. Zaitsev, N.Yu. Gordeev, V.M. Ustinov, P.S. Kop'ev. Jpn. J. Appl. Phys., 38 (2A), 674 (1999).

[23] M. Gurioli, A. Vinattieri, M. Colocci, C. Deparis, J. Massies, G. Neu, A. Bosacchi, S. Franchi. Phys. Rev. B, 44 (7), 3115 (1991).

Редактор Г.А. Оганесян

\section{Time-resolved photoluminescence of InGaAs nanostructures of different dimensionality}

\author{
A.M. Nadtochiy', S.A. Mintairov ${ }^{2}$, N.A. Kalyuzhnyy ${ }^{2}$, \\ M.V. Maximov ${ }^{1}$, D.A. Sannikov ${ }^{3,4}$, \\ T.F. Yagafarov ${ }^{3}$, A.E. Zhukov ${ }^{1}$ \\ ${ }^{1}$ St. Petersburg Academic University, \\ 194021 St. Petersburg, Russia \\ 2 loffe Institute, \\ 194021 St. Petersburg, Russia \\ ${ }^{3}$ Skolkovo Institute of Science and Technology, \\ 121205 Moscow, Russia \\ ${ }^{4}$ P.N. Lebedev Physical Institute, \\ Russian Academy of Sciences, \\ 119333 Moscow, Russia
}

\begin{abstract}
By using time-correlated single-photon counting timeresolved photoluminescence of quantum-sized heterostructures of different dimensionality was investigated. InGaAs quantum dots, quantum well, and transitionally-dimensional structure - quantum well-dots were grown on GaAs substrates. It was observed, that photoluminescence decay strongly depends on structure dimensionality resulting in decay value of 6,7 , and more than $20 \mathrm{~ns}$ for quantum dots, well-dots and well, respectively. As we believe localization centers in heterostructures may be responsible for such shortening of photoluminescence lifetime.
\end{abstract}

